\title{
Provenance in bioinformatics workflows
}

\author{
Renato de Paula ${ }^{1}$, Maristela Holanda ${ }^{*}$, Luciana SA Gomes², Sergio Lifschitz², Maria Emilia MT Walter ${ }^{1}$ \\ From The Second Workshop on Data Mining of Next-Generation Sequencing in conjunction with the 2012 \\ IEEE International Conference on Bioinformatics and Biomedicine \\ Philadelphia, PA, USA. 4-7 October 2012
}

\begin{abstract}
In this work, we used the PROV-DM model to manage data provenance in workflows of genome projects. This provenance model allows the storage of details of one workflow execution, e.g., raw and produced data and computational tools, their versions and parameters. Using this model, biologists can access details of one particular execution of a workflow, compare results produced by different executions, and plan new experiments more efficiently. In addition to this, a provenance simulator was created, which facilitates the inclusion of provenance data of one genome project workflow execution. Finally, we discuss one case study, which aims to identify genes involved in specific metabolic pathways of Bacillus cereus, as well as to compare this isolate with other phylogenetic related bacteria from the Bacillus group. B. cereus is an extremophilic bacteria, collected in warm water in the Midwestern Region of Brazil, its DNA samples having been sequenced with an NGS machine.
\end{abstract}

\section{Introduction}

The speed and efficiency with which scientific workflows may be performed have increased with the use of modern hardware and software technologies. One workflow can be executed many times with different programs, versions or parameters, or even modified input data, and scientists can compare results from these executions, which improves accuracy in data analysis. However, dealing with large volumes of information produced by many executions under a variety of conditions becomes increasingly difficult. In this context, new tools have to be developed to store data generated in each execution, together with the origin of this data and the details of a particular execution.

Therefore, on the one hand, data provenance is essential to scientific environments. Many works describe details of data provenance $[1,2]$, classify their characteristics [3], propose models [4-7] and present practical applications [8,9]. Buneman et al. [10] define data provenance as "the description of the origins of a piece of data and the process by which it arrived in a database". In other words, the origin of data used as raw material as well as the processes that transformed this data into the final

\footnotetext{
* Correspondence: mholanda@cic.unb.br

'Department of Computer Science, University of Brasilia - UnB, Brasilia, Brazil Full list of author information is available at the end of the article
}

product, must be identified and stored. It is important to note that, before choosing which data has to be stored, it is necessary to define how these data have to be structured so that they can be later recovered and understood. This explains the development of many distinct provenance models, e.g., W7 Model [4], Provenir Ontology [11], Provenance Vocabulary [5], Open Provenance Model (OPM) [6] and PROV-DM model [7].

On the other hand, genome projects aim to analyze DNA or RNA sequences produced by one NGS sequencer, e.g., 454/Roche [12]. According to the objectives of each project, many databases and programs are sequentially executed in a computational workflow, using DNA or RNA sequences as raw data and producing a large volume of information (Terabytes of data). Therefore, biologists analyze the generated data and, in order to obtain better results, propose improvements to the project, choosing different programs or requiring new executions of the same programs with different parameters. Thus, when one workflow execution is concluded, much information can be lost if there is no systematic procedure exists to store details of each execution, including adopted software and parameters, problems and related solutions, as well as information produced at each workflow step. 
In this study, we propose to manage data provenance in bioinformatics workflows using the PROV-DM model [7]. This provenance model aims to store details of each workflow execution in a way that biologists can compare information generated among different executions and more efficiently plan new ones. Stored data includes raw and produced data at each workflow step, used parameters, the order in which the programs are executed and details of how data are linked. Our provenance model allows us to recover the process flow as well as to reconstruct the relations among input data and the processes generating new data, using different granularities.

\section{PROV-DM model}

The PROV-DM model [7], proposed by the W3C (World Wide Web Consortium), is a conceptual data model that forms the basis for the W3C provenance (PROV) family of specifications. PROV-DM is based on the Open Provenance Model (OPM) [6] although more precisely defined. A PROV-DM showing the provenance description is graphically illustrated by a directed graph, rooted at the entity for which we want to explain the provenance.

PROV-DM core structure defines two initial elements, Activity and Entity, which can originate nodes in the provenance graph. Activity represents a process indicating the origin of one provenance object. Entity models any object representing some type of provenance. Entity element has two subtypes. The first one, Agent, can act over an Activity, or it presents some kind of responsibility over an Entity, e.g., owner or author's rights. The second one, Collection, represents a set of Entities, each one independent of its provenance content. Symbols representing elements of the provenance graph are illustrated in Figure 1.

Relations among the nodes are represented by the PROV-DM graph edges, which describesdifferent types of relations, the following ones being the most important to this study:

- wasDerivedFrom indicates that an original Entity was used, directly or indirectly, to generate another derived Entity;

- wasQuotedFrom is a particular type of wasDerivedFrom relation, and indicates that an Entity was generated from copying a part of an original Entity;

- used indicates that an Entity was used by an Activity;

- wasGeneratedBy indicates that an Entity was generated by an Activity;

- memberOf indicates which Entities belong to a Collection;

- wasAssociatedWith indicates that an Activity was associated to an Agent.

Constraint is another important PROV-DM property. Constraints are related to rules or restrictions associated with the construction of a provenance graph, having

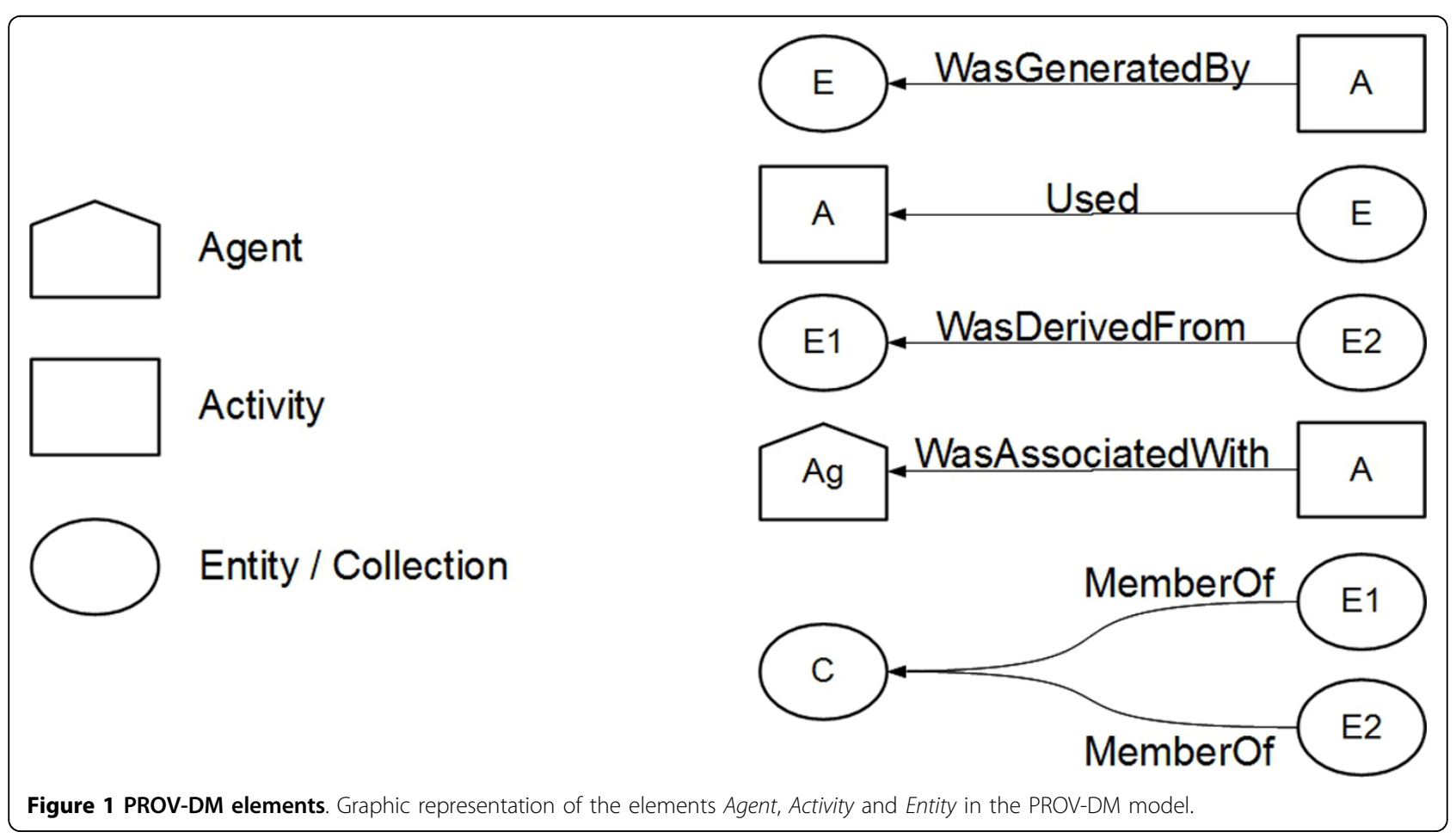


two main objectives: preventing the creation of invalid or inconsistent graphs; and making inferences about the elements and the relationships among them.

Besides this, PROV-DM annotation allows one to write additional information to any element of a provenance graph, which is extremely important since details about a specific process or element in a particular execution of a workflow can then be stored.

\section{Genome project}

High-throughput sequencing machines, e.g., 454/Roche [12], produce hundreds of thousands of DNA or RNA fragments that have to be analyzed in genome or transcriptome projects. These NGS sequencers generate Gigabyte files, containing DNA or RNA sequences to be processed in computational systems named "workflows". Common phases in a workflow are described next.

In the filtering phase, the sequences are "cleaned" such that only the ones with a minimum of quality are included in the following phases, i.e., low quality sequences are discarded. This is possible because NGS sequencers provide quality for each base (represented as a letter in the alphabet $\Sigma=\{A, C, G, T$ (DNA) or $U$ (RNA) $\}$ ) of each fragment (a string in $\Sigma$ ). Data format generated by the sequencer is modified to string files, which will be processed in the following phases.

In the assembly phase, groups of fragments sharing similar regions are clustered into contigs. This is done for large fragments, such as those generated by $454 /$ Roche. Longer regions containing contigs form scaffolds, constructed using a phylogenetic related organism as reference genome.

In the analysis phase, according to the objectives of the genome or transcriptome project, different analyses may be executed, e.g., annotation (a biological function is assigned to each contig, using comparison algorithms and large databases), identification of enzymes in order to find putative metabolic pathways involved in certain reactions, or phylogenetic reconstruction showing evolutionary relationships among the studied and related organisms. The analysis phase is the most space expensive one, since it may generate Terabytes of information, when compared to the previous phases, which generate Gigabytes of information.

In a genome or a transcriptome project, information may be stored in a file system or a DBMS. Input (raw data) for the workflow is the set of sequences generated by one NGS sequencer. As previously mentioned, outputs depend on each project objectives, but each workflow phase deals with specific databases and programs generating very large amounts of data. This information, together with versions and parameters of the programs, must be stored. Moreover, genome and transcriptome projects usually involve different executions of the same workflow using different parameters or including other databases. In this context, a provenance model is very important since it guarantees access to details of each execution of the workflow, and this allows the biologists to study how the modifications can affect the resulting data, so improving the analyses.

\section{Data provenance in genome projects}

Managing data provenance in genome projects is essential to control how the enormous volumes of data were generated since, as mentioned earlier, they involve distinct analyses performed by many programs and data bases, with different versions and parameters. We propose here to use the PROV-DM model to manage data provenance in bioinformatics workflows. The PROV-DM model is simple, well documented, and can be easily adapted to include provenance in genome workflows [13].

We propose a model with two levels of granularity, in order to keep structured bioinformatics provenance information. The first level corresponds to the PROV-DM model based provenance graph, and includes details about each graph node. The graph is composed by Collections, performed Activities and Agents operating in a particular execution, besides presenting relationships between each pair of elements. Collections are used to represent a group of Entities, which are composed of millions of DNA or RNA sequences.

Relations in our PROV-DM based model are:

- memberOf linking Entities to Collections;

- Used and WasGeneratedBy maintain their behavior when Collection is used;

- WasDerivedFrom characterizes a transformation of an Entity or a Collection into another.

To represent data provenance in a bioinformatics workflow, we propose to include two new elements, Project and Workflow Execution, besides the PROV-DM components. Project was created to join distinct re-executions of a genome project workflow. Workflow Execution stores information about one workflow execution. A set of minimum information related to each entity is shown in Table 1.

The second level allows access to the original input files used in one workflow execution. e.g., transcripts (expressed genes), as well as the output files produced in each phase of the workflow. Since one execution of a workflow generates large amounts of information, which may, or may not, be relevant for future analysis, biologists can decide what information will be stored or discarded.

Biologists can choose which levels will be maintained. The first level needs only a few Bytes of memory since it requests little information, in contrast to the second level that may require Terabytes of space, depending on 
Table 1 Information of the provenance graph for a genome project (already shown in de Paula [16])

\begin{tabular}{|c|c|c|c|}
\hline \multicolumn{2}{|r|}{ PROJECT } & \multicolumn{2}{|r|}{ WORKFLOW EXECUTION } \\
\hline $\begin{array}{l}\text { Name } \\
\text { Description } \\
\text { Funding Institutions } \\
\text { Partner Institutions } \\
\text { Coordinator } \\
\text { Start Date } \\
\text { End Date }\end{array}$ & $\begin{array}{l}\text { Name of the Project } \\
\text { Description of the Project } \\
\text { List of the Project funding institutions } \\
\text { List of the Project partner institutions } \\
\text { Name of the Project coordinator } \\
\text { Project Start date } \\
\text { Project End date }\end{array}$ & $\begin{array}{l}\text { Name } \\
\text { Description } \\
\text { Location } \\
\text { Date } \\
\text { Version Date } \\
\text { Version } \\
\text { Notes }\end{array}$ & $\begin{array}{l}\text { Name of the Workflow Execution } \\
\text { Description of the Workflow Execution } \\
\text { Execution Location } \\
\text { Execution Date } \\
\text { Version Date } \\
\text { Version number } \\
\text { Any information about the execution of } \\
\text { the Workflow Execution }\end{array}$ \\
\hline \multicolumn{2}{|r|}{ AGENT } & \multicolumn{2}{|r|}{ ACTIVITY } \\
\hline $\begin{array}{l}\text { Name } \\
\text { Institution } \\
\text { Position } \\
\text { Function } \\
\text { Groups } \\
\text { Notes }\end{array}$ & $\begin{array}{l}\text { Name of the person } \\
\text { Affiliated Institution } \\
\text { Position or Function } \\
\text { Function in the Workflow Execution } \\
\text { Filtering provenance graph groups } \\
\text { Any information about Agent }\end{array}$ & $\begin{array}{l}\text { Name } \\
\text { Program } \\
\text { Version } \\
\text { Command Line } \\
\text { Function } \\
\text { Start Time } \\
\text { End Time } \\
\text { Environment } \\
\text { Groups } \\
\text { Notes }\end{array}$ & $\begin{array}{l}\text { Name of the Activity } \\
\text { Program Name } \\
\text { Program Version } \\
\text { Command Line used and parameters } \\
\text { Description of what is being done } \\
\text { Date and time the Activity began } \\
\text { Date and time the Activity ended } \\
\text { Description of computational } \\
\text { environment } \\
\text { Filtering provenance graph groups } \\
\text { Any information about the execution of } \\
\text { the Activity }\end{array}$ \\
\hline \multicolumn{2}{|r|}{ COLLECTION } & \multicolumn{2}{|r|}{ ENTITY } \\
\hline $\begin{array}{l}\text { Name } \\
\text { Size } \\
\text { Description } \\
\text { Location } \\
\text { Group } \\
\text { Notes }\end{array}$ & $\begin{array}{l}\text { Name of the Collection } \\
\text { Number of Entities contained in Collection } \\
\text { Description of the Collection content } \\
\text { Location of the file or database with the } \\
\text { Content of the Collection } \\
\text { Filtering provenance graph groups } \\
\text { Any information about the content of the Collection }\end{array}$ & $\begin{array}{l}\text { Name } \\
\text { Description } \\
\text { Location } \\
\text { Group } \\
\text { Notes }\end{array}$ & $\begin{array}{l}\text { Name of the Collection } \\
\text { Description of the Entity content } \\
\text { Location of the file or database with the } \\
\text { content of the Entity } \\
\text { Filtering provenance graph groups } \\
\text { Any information about the content of the } \\
\text { Entity }\end{array}$ \\
\hline
\end{tabular}

the genome project objectives. Although separated, information of the two levels remain linked, which allows transparent access to these data.

\section{PROV-DM for provenance in bioinformatics workflows}

PROV-DM model defines elements and relationships, as well as restrictions and inferences that can be used in a variety of ways according to the context in which data provenance is applied. In this section, these topics are discussed with regard to bioinformatics workflows.

\section{Managing elements}

The PROV-DM elements used in this project are entity, activity, agent and collection, which represent possible nodes of the provenance graph, and account to represent the graph itself. In the following, we discuss how we used them in our proposal of a provenance model for bioinformatics workflows.

We first address the entity element, which has three types: agent, account and collection. The agent element will be used to represent any person, institution or service that makes some kind of action in an activity. The account element will capture the execution of a particular experiment, and therefore it represents a provenance graph. The entity element will be used to represent basic data, e.g., a DNA sequence or an alignment. When a workflow is executed in a genome project, the entity element will be used when the user needs to know the content of a collection. It is always possible to graphically exhibit all the entities, but since some information, such as the raw data, wastes large amounts of storage space, sometimes it is not possible to show all of them, but only the collections themselves.

The other basic PROV-DM element is activity. In bioinformatics projects, an activity represents any process that can be executed in an experiment (workflow). The activity element will indicate the properties of one executed program, including command lines and program name and version, among others. Notably, among the elements composing the graph (entity, agent, collection and activity), the later is the only one having temporal characteristics (start and end times).

Finally, with the objective of improving the creation of personalized provenance graphs based on distinct executions of a workflow, an attribute called Group was defined. This attribute can be found in the nodes of the graph or in the collection elements. A group is defined by the user when each element is created and, due to its multiple attribute status, each element can be part of different groups. This allows the user to visualize only a part of the provenance graph by choosing some of these groups. 


\section{Managing relations}

PROV-DM relations are represented by edges linking different nodes of the provenance graph. These edges are directed and show how each object was generated, since the path in the graph begins in the generated object and comes to the origin of this data, passing through the events that generated that object. The PROV-DM model describes different types of relations, and since we are working on bioinformatics projects, we have adopted some definitions.

The first relation to be considered is Used, which links a collection or an entity to the activity that used this collection or entity. A directed edge goes from the activity to a collection or entity, which can be used in distinct activities. Similarly, each activity can use different collections or entities. This is good for bioinformatics projects, since a particular file can be used by different processes, e.g., a genome reference can be used in different mapping processes.

The relation WasGeneratedBy indicates which activity generated a particular collection or entity. The edge goes from the collection or entity to the activity that generated it. One activity can generate a variety of collections or activities, however each collection or entity can only be generated by a single activity. This way, different WasGeneratedBy relationships can be created for only one activity, however, only one activity can occur for each collection or entity. This definition models the processes executed in bioinformatics projects, where one particular data (or file) can only be generated by a single program.

The relation WasAssociatedWith stores the agent that has executed an action of an activity. The edge goes from the agent to the activity. Multiple connections among different agents and activities are allowed.

The relation WasDerivedFrom indicates how the link was derived, during the execution of one experiment, between the used and the generated data. In a bioinformatics workflow, different processes can be executed, so the attribute Type of Derived Link was associated to each edge with one of the following characteristics: filtering, the collection was derived by a filtering process; ordering, the derived collection was rearranged according to some ordering criterion; mixing, the created collection has a data format different from the original one; other, any other process distinct from the previous ones.

Considering that the relations Used, WasGeneratedBy, WasAssociatedWith and WasDerivedFrom can present multiple links, and when the identification of these links store important provenance information in the graph, the attribute Role provides the identification of each one of these links. This way, each of the four relationships has an associated attribute Role, which value will be defined by the user to precisely indicate the reason why the corresponding edge has been created.

\section{Restrictions}

As previously stated, PROV-DM model defines a list of generic restrictions in order to allow the validation of the provenance graph. These restrictions are divided into three types: structural, temporal and functional, described as follows.

\section{Structural restrictions}

Structural restrictions allow the construction of a more concise provenance graph, avoiding ambiguous information and guaranteeing that the execution of each experiment (one execution of the bioinformatics workflow) can be reproduced. The most important restriction for keeping the provenance graph structured is that each element of the graph has to have a single identifier, including the graph itself. This also supports the creation of relationships using the identification of each element.

Another structural restriction is the creation of valid relationships in the provenance graph. A single relationship can be created from existing and valid nodes. Existing nodes are those that have already been created and inserted in the experiment, and valid nodes are those expected for each relationship.

These relationships allow the construction of the provenance graph structure. Thus, we defined a list of restrictions that must be followed when creating a relationship:

- no two identical relationships exist, i.e., it is not possible to have two relationships of the same type with the same origin and the same destination;

- only one relationship WasGeneratedBy linked to each entity or collection of the provenance graph is allowed;

- in the relationship WasDerivedFrom, the elements "origin" and "destination" must be different.

\section{Temporal restrictions}

The objective of temporal restrictions is to avoid creating graphs representing sequences of processes that are impossible to occur in a bioinformatics workflow. To simplify the creation of the bioinformatics workflow provenance graph, we defined three elements with temporal characteristics: project, account (representing the graph itself) and activity.

An activity models the execution of some process having a start time and an end time. This characteristic is passed to the other elements as follows: WasGeneratedBy, the entities and collections will have the hour in which the generated activity was completely concluded as the start time; Used, the entities and collections will have as their start times the hours that the used activity began as 
their start times; WasAssociatedWith, the agent activity times are the activity start time and end time.

These characteristics allowed us to adopt the following restrictions:

- Activity: the start time of an activity must be less than its end time;

- WasGeneratedBy: a collection or an entity can only be generated by an activity whose end time is less than all the hours when this collection or entity has begun to be used;

- Used: analogously, an activity can only use a collection or entity if the start time of this activity is greater than the start time of this collection or entity;

- WasDerivedFrom: a collection or entity can only be derived from another original collection or entity if the start time of the original is less than the hour of having generated the derived one.

Temporal restrictions of Used, WasGeneratedBy and WasDerivedFrom can only be evaluated when the elements that can be validated are identified, which means that the restrictions can only be observed from the moment that there exists at least one collection or one entity being used by an activity and being generated by another activity exists. This is a minimum set of temporal restrictions that need to be verified. Figure 2 shows an example of a graph having a minimum set of elements that allows the verification of these three temporal restrictions, since the temporal characteristics (start and end times of each activity) need to be validated.

\section{Functional restrictions}

The last type of restrictions refers to the functionality of an experiment. Collections point to sets of data that are usually files containing information used and generated during the execution of this experiment. This restriction refers to the correct identification of these collections. Thus, collections can only indicate sets of correct data, or rather, indicate a file that has to have its existence verified.

\section{Inferences}

Inferences allow the verification of some Roles. In our provenance model, some inferences were determined from observing some restrictions described in the previous section.

The first inference is related to the structural validity of an account, which can be verified from the account structural restrictions. Once all of these structural restrictions are verified, one can infer that the account is structurally valid. Otherwise, this account can be inferred as structurally invalid. Analogously, the structural validity of a project can also be inferred. Therefore, if all the accounts belonging to a project are structurally valid, then this project is also inferred as structurally valid. If it is not the case, even if only one account is structurally invalid, the project is considered structurally invalid.

The temporal inference analyzes temporal characteristics of the account. Thus, we can verify those related to the account start and end times. If all these restrictions are correct, the other two situations related to the activity temporal restrictions must be followed. The temporal restrictions referring to Used, WasGeneratedBy and WasDerivedFrom relationships will only be possible if there is a minimum set of nodes and edges. For accounts not having a minimum set, this restriction will be automatically satisfied. Therefore, for an account to be inferred as temporally valid, the temporal restrictions of their start and end times have to be satisfied, as well as the temporal restrictions of its activities and edges. Otherwise, it is considered temporally invalid. A project,

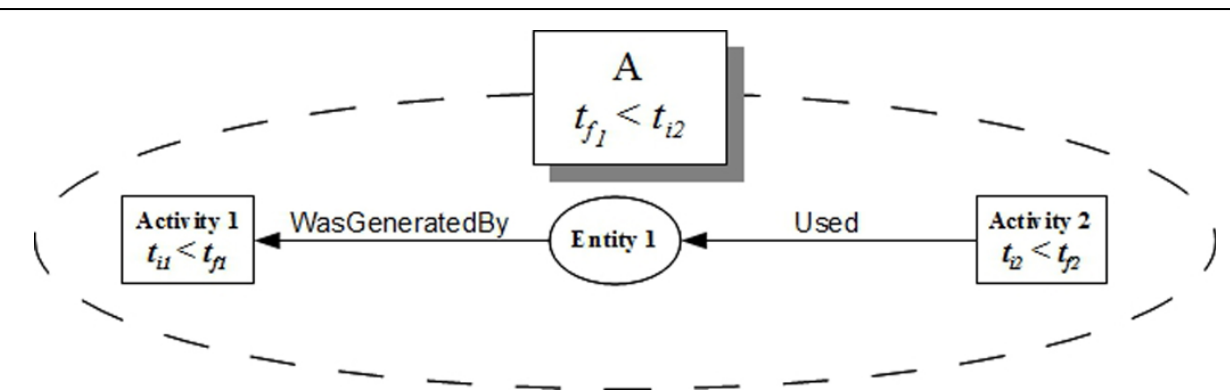

Figure 2 A graph with a minimum set of elements allowing the verification of temporal restrictions. Besides the activity element, two other elements have temporal characteristics, account and project. Both elements work only with the start time and end time. This way, four more restrictions can be defined: Account: the start time of an account must be less than or equal to the end time; Account-Activity: an account can contain only activities executed between start time and its own execution end times; Project: the start time of a project must be less than or equal to the end time; Project-Account: a project can only contains accounts representing experiments executed between the project start and end times. 
in its turn, can be inferred as temporally valid if the restrictions related to its start and end times are satisfied, and all the accounts that belong to this project are temporally valid. If this is not the case, the project is inferred as temporally invalid.

The functional inferences are related to the functional restrictions, thus one can infer that an experiment is functionally valid if all its collections point to sets of valid data. Otherwise, it will be inferred as functionally invalid. An experiment considered functionally invalid is not necessarily treated as an experiment containing errors, but it means that at least one of its collections points to some non-available data set. The user can choose to maintain or not some files used in an experiment and, if a file is deleted from this experiment, it will be functionally invalid. Even though it may not be considered an error, the evaluation of the functional validity is important, since it means that this experiment cannot be reexecuted due to a possible lack of necessary elements. Analogously, a project will be considered functionally valid if all of its experiments are functionally valid. If one experiment is considered functionally invalid, then the entire project is considered functionally invalid.

\section{Related work}

Some projects in the literature propose to store provenance data in Database Management Systems (DBMS). The DBMS was used in the proposals of Jones et al. [14], Mungall and Emmert [15] and Paula et al. [16], which created biological databases with specific modules to include provenance data. However, the DBMS table structure is inflexible to store the different types of genome projects data.

Other projects use the W7 model. Marins et al. [17] present an application to find information in personal computers, and catalog them to facilitate their research. Liu and Ram [18] use provenance data to evaluate the quality of Wikipedia pages based on the ways users collaborate. Orlandi et al. [9] present a model using pages and categories for provenance capture in Wikipedia pages to show user contribution information. The Provenance Vocabulary model [19] presents an application that uses provenance data on the web for evaluating the quality of available information. Omitola et al. [20] extends this model to integrate web data. Kessler et al. [21] treat provenance data in OpenStreetMap using the Provenance Vocabulary model.

Provenir Ontology [22] extends a model designed to deal with biological data in a project having the objectives of developing a vaccine, a diagnostic exam and a chemotherapy treatment for the Trypanossoma cruzi human pathogen. Missier and Belhajjame [23] use the Provenir Ontology model together with a module for treating biological data, addressing the data collection generated by Taverna (one Scientific Workflow Management System - SWfMS) [24]. As an example, they discuss how to search known relationships between a specific region in the mouse genome, known as QTLO (Quantitative Trait Loci), and the metabolic pathways involving genes located in this region. Patni et al. [25] present a framework to store and query provenance data in meteorological data.

OPM is the most widely used model in applications of provenance now, as can be seen by the variety of projects using it. Here we discuss some of them. Cao et al. [26] present an OPM based system to capture and generate provenance data in scientific experiments. Marinho et al. [27] and Coutinho et al. [28] aim at managing provenance information in heterogeneous and distributed environments using OPM. Chapman et al. [29] define an OPM based model to evaluate data reliability using provenance information. Braun et al. [30] evaluate the interoperability of provenance data among systems PASS (Harvard Provenance Aware Storage System) and MITRE's PLUS. Gomes [31,32] defines an OPM based system for capturing provenance data from biological workflows using SWfMS, storing these data in a database modelled according to the OPM model.

As far as we know, there are no applications based on PROV-DM for bioinformatics applications, since it is a very recent model. However, it is noteworthy that Missier and Belhajjame [33] present an application of deduction rules to verify the validity of provenance graphs.

Our model was designed to store and manage provenance data independent of any SWfMS, e.g., ad-hoc projects. In fact, little additional information is included in the original files in order to retrieve information using specific modules directly linking biological data types in these files. Our model does not address automatic capture of provenance data.

\section{Case study}

In this section, we first describe our Provenance simulator, and subsequently discuss one case study.

\section{The Provenance simulator}

A Provenance simulator was implemented in Java (details shown in de Paula [16]). We used two external libraries. The first one, OPM4J [6], was used to create an OPM based provenance graph. This library was used due to its stability, and to the fact that PROV-DM and OPM are very similar, and PROV-DM does not have completely developed libraries yet. The second one, GraphViz [34], was used to visualize the created provenance graph.

The simulator has a graphic interface allowing the user to inform provenance data, besides storing data in XML files. The user can create or visualize the provenance 
graph at any time, when a workflow is executed. The elements of the XML schema correspond to the information described in Table 1, created for each Project. Besides, a list was generated with the name and location of the XML files representing each Workflow Execution performed in a particular Project.

The Provenance simulator is shown in Figure 3, where the case study Multiple Alignment is displayed using information of an XML file previously stored. To use this simulator, the user needs to create a project, enter provenance data (Table 1), and inform the graph nodes (Agent, Activity and Entity) and relations linking these nodes (Figure 4). Data is stored in XML files, noting that, whenever this file is saved, the simulator modifies the file version and date, and a gif format file containing the provenance graph is generated. The simulator allows to visualize the provenance graph, as shown in Figure 5.

\section{The project: Identifying metabolic pathways of alpha-} amylases in a bacterial isolate

This case study deals with the DNA sequencing of the extremophilic bacteria Bacillus cereus, of which its isolate was collected in warm water in a city of the MidWest Region of Brazil. The objective of this project is to find the genes codifying for the alpha-amylase (obtained from UNIPROT [35]) in the B. cereus genome, as well as to compare their sequences with others belonging to related species in the Bacillus group. In this case study, we modeled data provenance of the comparisons among the sequences found in this isolate with genes of the related organisms. These comparisons were done using multiple alignments carried out for samples of the isolate alpha-amylase families 13 and 57, and drawing up graphics for these alignments. This project is under development in the Molecular Biology Laboratory of the Department of Cellular Biology at the University of Brasilia (UnB). Figure 6 shows the workflow of this experiment, and Figure 5 shows the provenance graph generated from one execution of the workflow.

This experiment was executed by a student of the Department of Computer Science at UnB, modeled by the agent AG001_Joao, and was validated by a researcher from the Department of Cellular Biology, represented by the agent C002_Taina. Agent AG001_Joao is associated

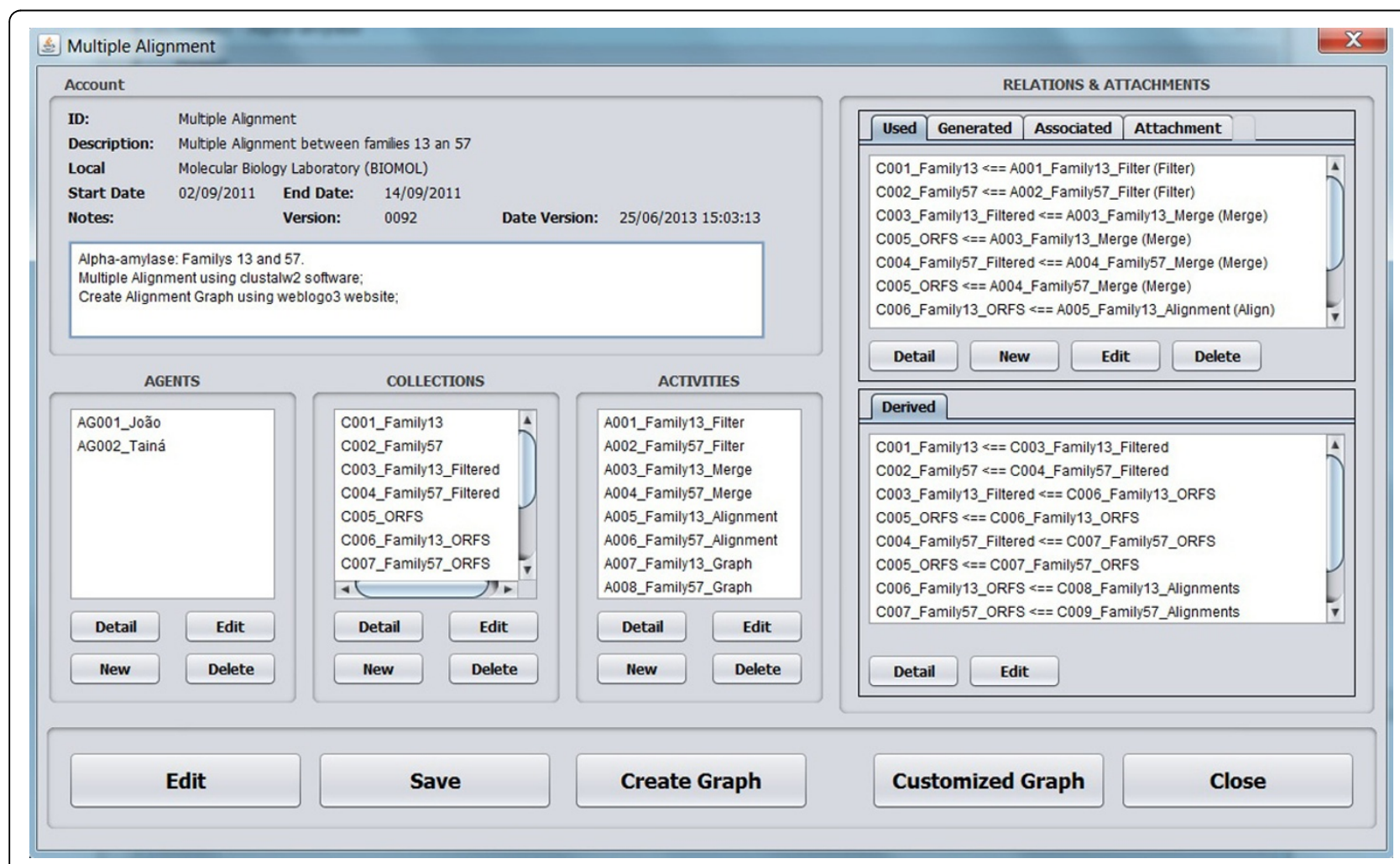

Figure 3 Main frame of the Provenance simulator. In the main frame of the simulator, called Provenance, a user can enter information to store data provenance for each execution of a particular bioinformatics workflow. In the upper left frame, general information of the execution (description and execution date) can be seen. Besides, elements Agent, Entity and Activity are listed, together with the three types of relations and the annotations called Attachments. With the objective of making it user friendly, Collections were implemented as Entities with "Size" property greater than 1. Finally, a relation WasDerivedFrom is found on the lower right frame, since it cannot be modified by users. Its creation or removal is originated from the creation/deletion of the relations Used and WasGeneratedBy. 


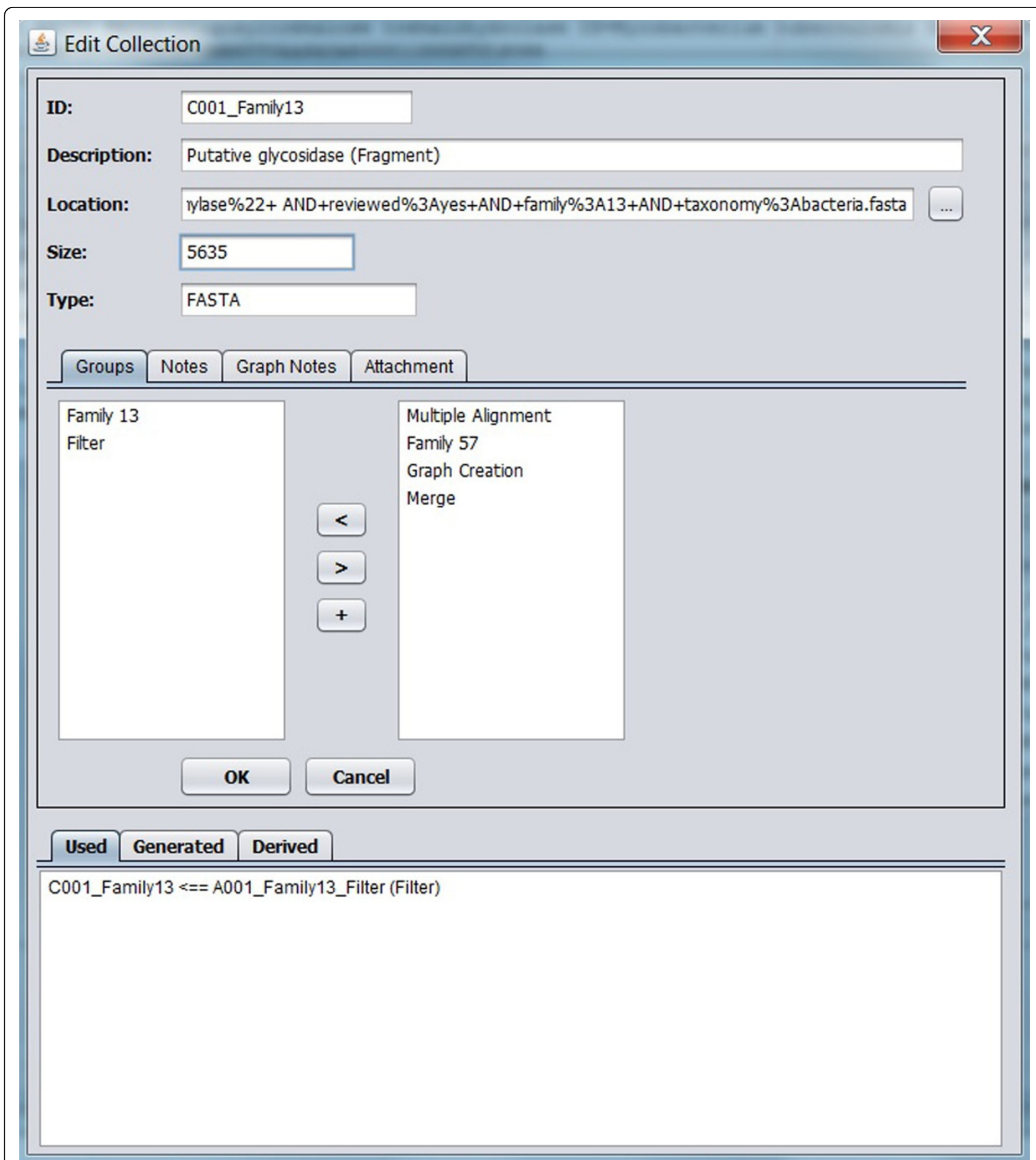

Figure 4 Sample of Entity edition frame. Data from C001_Family13, its groups and relations are shown in the frame data.

to all of the activities executed in this experiment, which is represented by the dotted edges between this agent and each activity on the graph. The edges represent the PROV-DM WasAssociatedWith relation. Along with this, the role of this agent was to execute each activity, which is shown by the Execution role present at each of its relationships. Agent C002_Taina validated four activities of the experiment, A005_Family13_Alignment, A006_Family57_Alignment, A007_Family13_Graphic and A008_Family57_Graphic, which is represented by the relationships among this agent and its activities, as well as by the role associated to each of these relationships. 

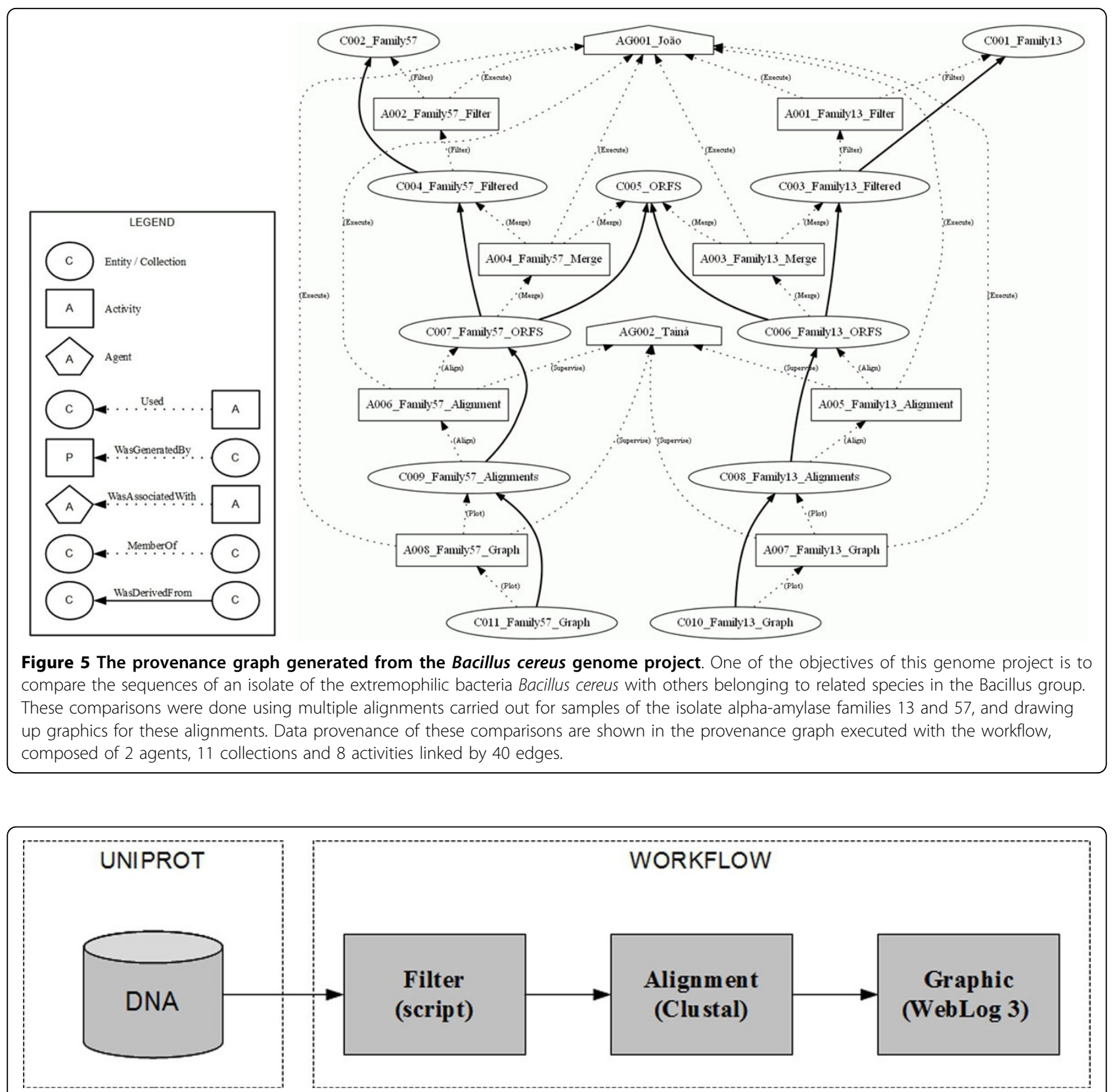

Figure 6 Workflow for finding alpha-amylase genes of a Brazilian Bacillus cereus isolate. The DNA sequences obtained from UNIPROT were first filtered and then aligned to a file containing the sequences of the alpha-amylase genes. Finally, the file with the multiple alignments was used to generate graphics.

Collections C0001_Family13 and C002_Family57 represent FASTA format files with the DNA sequences of families 13 and 57, respectively, of the alpha-amylases obtained from UNIPROT. Collection C0005_ORFS also represents a FASTA file of the putative proteins found on the contigs, which will comprise a part of the aligned ORFs in each family.

Initially, a filtering process was carried out, represented by activity A001_Family13_Filter, which used the original collection C001_Family13 to generate the filtered collection C003_Family13_Filtered. Next, the activity A003_Family13_Mix executed a process in order to join collections C003_Family13_Filtered and C005_ORFS, generating the collection C006_Family13_ORFS. After that, the collection C006_Family13_ORFS was used by the activity A005_Family13_Alignment with the purpose of arranging the multiple alignment of the sequences in this collection. These alignments were recorded in a file 


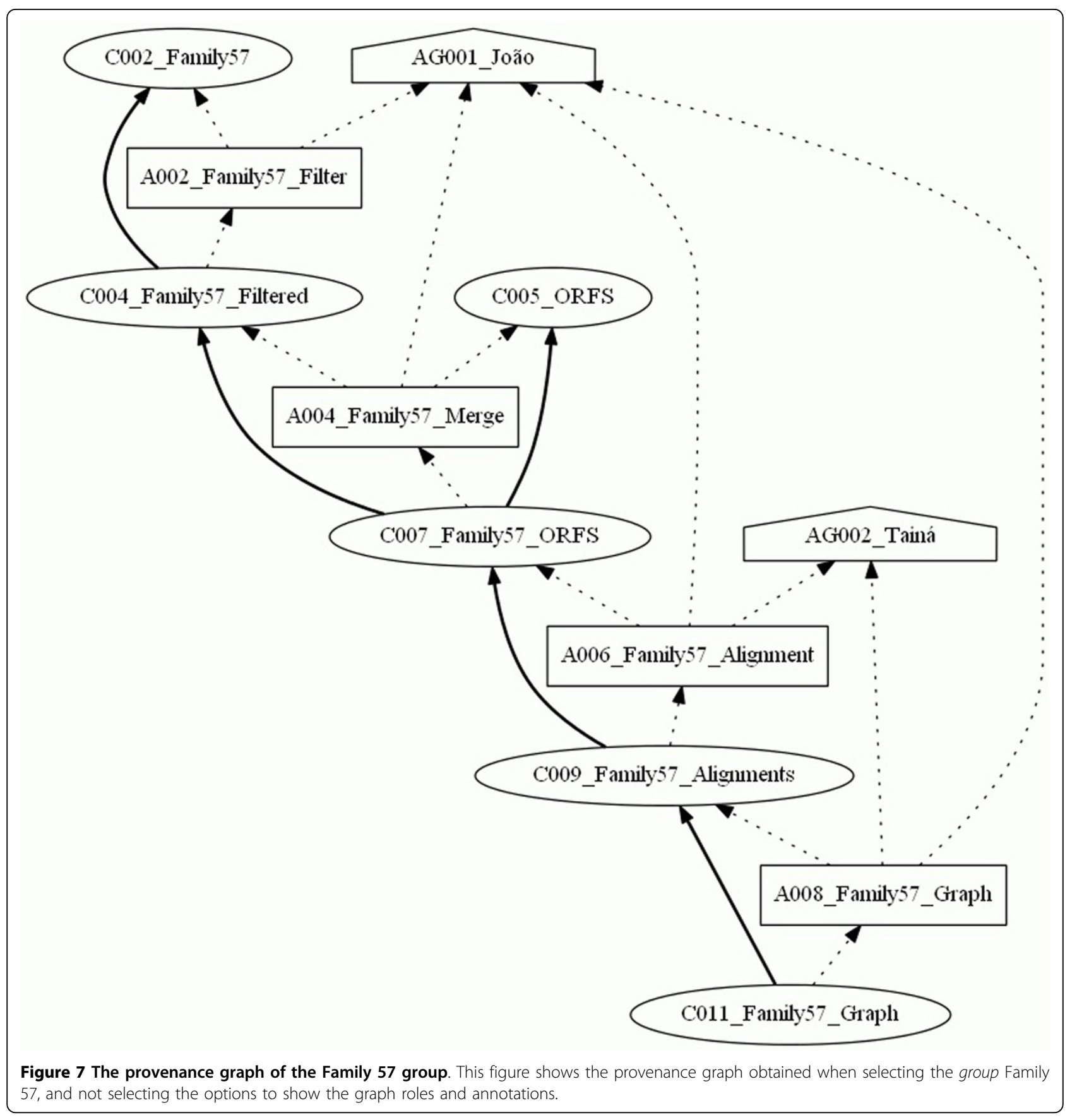

represented by the collection C008_Family13_Alignments. Finally, the collection C010_Family13_Graph was generated by the activity A007_Family13_Graph, which used the alignments contained in the collection C008_Family13_Alignments. The same processing steps occurred with the collections of Family 57, which generated the collection C011_Family57_Graph.

With the defined element group, it was possible to select parts of the experiments. In Figure 7, the group Family 57 was the only one selected, noting that the options to show the graph roles and annotations were not selected. In Figure 8, the provenance graph included the Multiple Alignment group and the option to present roles. Therefore, the graph shows only the nodes belonging to the Multiple Alignment group and their respective roles. Finally, a provenance graph was generated with nodes belonging to at least one of the groups, Filter or Multiple Alignment, together with an option to show the annotations. Figure 9 shows the graph with nodes belonging to at least one of the chosen groups, with the 


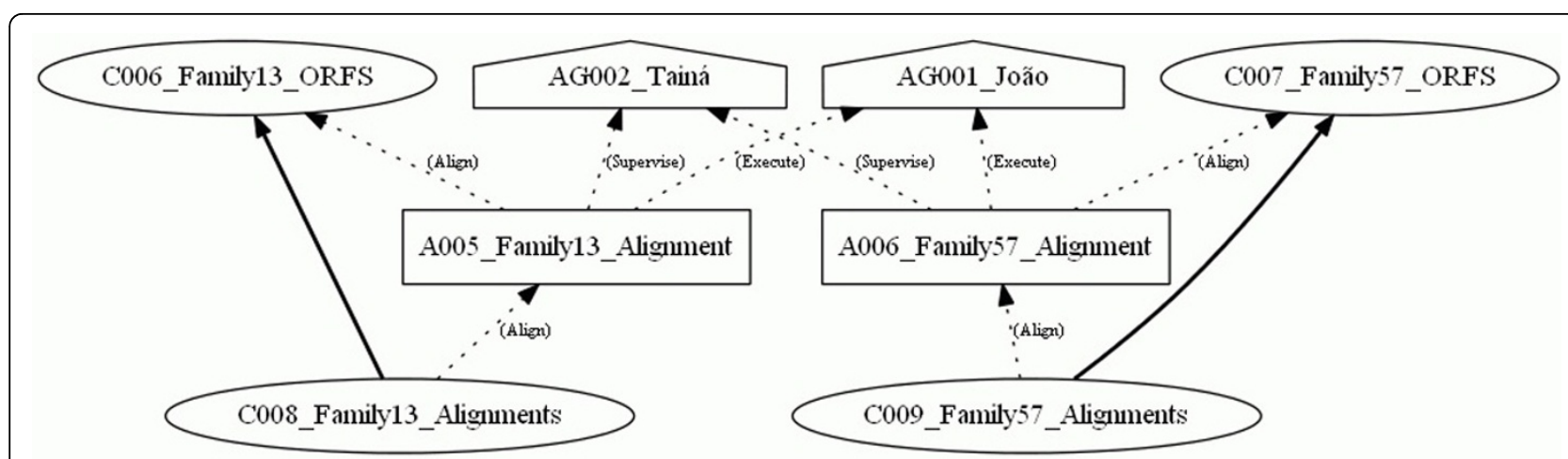

Figure 8 The provenance graph of the Multiple Alignment group and roles. This graph only shows the nodes belonging to the Multiple Alignment group and their respective roles.

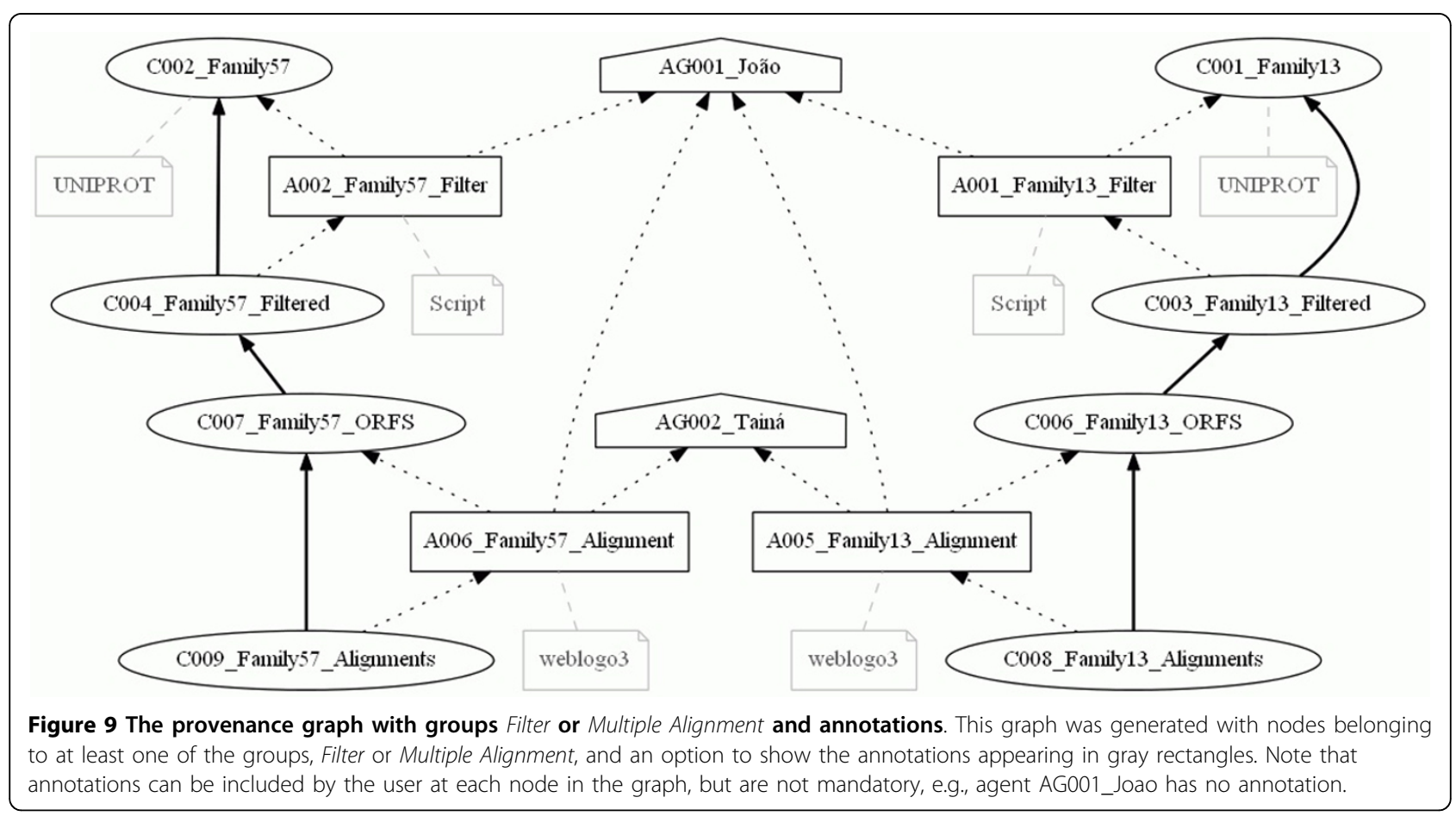

annotations indicating the origin of some of the collections and programs used for the activity executions.

\section{Conclusion}

In this work, we propose to use the PROV-DM model to manage data provenance in genome projects. The PROV-DM model allows to store the properties of each execution of a bioinformatics workflow. To graphically represent the large volumes of data generated at the genome projects, we used Collections of Entities to represent groups of large sets of data, and the same files storing the project data to construct the Collections. The proposed provenance model was divided in two levels, one corresponding to the provenance graph itself and the other providing access to the data of a particular execution. Biologists can investigate the entire or just a portion of the provenance graph.

We also developed one case study, including provenance in a bioinformatics workflow with real data. This experiment shows that little additional data has to be generated in order to maintain provenance. To create the provenance graph, we only need to connect data provenance to the files already used in a particular workflow execution. This case study was developed in order to show the usability of the provenance model in a real genome project, which had the objective of finding alpha-amylases in an isolate of an extremophilic bacillus collected in warm water in the MidWest 
Region of Brazil. We also created the Provenance simulator, for storing details of the workflow execution in XML files.

We are developing a module in the Provenance simulator to automatically capture provenance data from a workflow execution, with few information given by the user. Besides, it is useful to integrate our Provenance simulator in a bioinformatics workflow implemented using a SWfMS.

\section{Competing interests}

The authors declare that they have no competing interests.

\section{Authors' contributions}

R. P. implemented the Provenance simulator and developed the experiments. R. P., M. H. and S. L. discussed the experiments. All the authors proposed the provenance model and contributed to the text.

\section{Acknowledgements}

M.E.M.T.W. thanks CNPq (process 306731/2009-6) and FINEP (process 01.08.0166.00)

\section{Declaration}

The publication costs for this article were funded by the University of Brasilia.

This article has been published as part of BMC Bioinformatics Volume 14 Supplement 11, 2013: Selected articles from The Second Workshop on Data Mining of Next-Generation Sequencing in conjunction with the 2012 IEEE International Conference on Bioinformatics and Biomedicine. The full contents of the supplement are available online at http://www. biomedcentral.com/bmcbioinformatics/supplements/14/S11.

\section{Authors' details}

'Department of Computer Science, University of Brasilia - UnB, Brasilia, Brazil. ${ }^{2}$ Department of Informatics, Pontificial Catholic University - PUC/RJ, Rio de Janeiro, Brazil.

\section{Published: 4 November 2013}

\section{References}

1. Tan WC: Research problems in data provenance. IEEE Data Engineering Bulletin 2004, 27:45-52.

2. Cheney J: Workshop on theory and practice of provenance event report. SIGMOD Rec 2009, 38:57-60.

3. Glavic B, Dittrich K: Data Provenance: A Categorization of Existing Approaches. Verlagshaus Mainz, Aachen. 2007.

4. Ram S, Liu J: Active conceptual modeling of learning. In Active conceptual modeling of learning. Berlin, Heidelberg: Springer-Verlag;Chen PP, Wong LY 2007:17-29[http://portal.acm.org/citation.cfm?id=1793834.1793838].

5. Hartig O, Zhao J: Publishing and Consuming Provenance Metadata on the Web of Linked Data. Provenance and Annotation of Data and Processes 2010, 6378(24):78-90.

6. Moreau L, Clifford B, Freire J, Futrelle J, Gil Y, Groth P, Kwasnikowska N, Miles S, Missier P, Myers J, Plale B, Simmhan Y, Stephan E, den Bussche JV: The Open Provenance Model - Core Specification (v1.1). 2010 [http:// openprovenance.org/].

7. W3C: PROV-DM: The PROV Data Model. 2012 [http://www.w3.org/TR/prov$\mathrm{dm} / \mathrm{s}$.

8. Aldeco-Pérez R, Moreau L: Provenance-based Auditing of Private Data Use [http://eprints.ecs.soton.ac.uk/16580/, BCS 2008.

9. Orlandi F, Passant A, Champin PA: Semantic Representation of Provenance in Wikipedia. Second International Workshop on Role of Semantic Web in Provenance Management (SWPM 2010 - Workshop of ISWC 2010) 2010, 1-6[http://liris.cnrs.fr/publis/?id=5017].

10. Buneman P, Khanna S, chiew Tan W: Why and Where: A Characterization of Data Provenance. In ICDT Springer; 2001, 316-330
11. Sahoo SS, Sheth A: Provenir ontology: Towards a Framework for eScience Provenance Management. Microsoft eScience Workshop 2009, 1.

12. Rothberg JM, Leamon JH: The development and impact of 454 sequencing. Nature Biotechnology 2008, 26(10):1117-1124.

13. Paula R, Holanda MT, Walter MEMT, Lifschitz S: Managing data provenance in genome project workflows. Bioinformatics and Biomedicine Workshops (BIBMW), 2012 IEEE International Conference on: 4-7 October 2012 2012, 654-661.

14. Jones A, Hunt E, Wastling JM, Pizarro A, Stoeckert CJ: An object model and database for functional genomics. Bioinformatics 2004, 20(10):1583-1590 [http://eprints.gla.ac.uk/21950/].

15. Mungall CJ, mEmert DB: A Chado case study: an ontology-based modular schema for representing genome-associated biological information. Bioinformatics 2007, 23(13):i337-i346[http://www.ncbi.nlm.nih.gov/pubmed/ 17646315]

16. de Paula R, Huacarpuma RC, Holanda MT, Walter MEMT: A Provenance Data Model to Manage RNA-SEQ Projects. Proceedings of 4th International Conference on Bioinformatics and Computational Biology 2012 2012, 165-170.

17. Marins A, Casanova MA, Furtado A, Breitman K: Modeling Provenance for Semantic Desktop Applications. Proceedings of the XXVII Congresso da SBC - XXXIV Semin'ario Integrado de Software e Hardware 2007, 2100-2012.

18. Liu J, Ram S: Who Does What: Collaboration Patterns in the Wikipedia and Their Impact on Data Quality. Proceedings of nineteenth Annual Workshop on Information Technologies and Systems (WITS 2009) Phoenix, Arizona, USA; 2009, 1-8.

19. Hartig O, Zhao J: Using Web Data Provenance for Quality Assessment. SWPM 2009, 1-6.

20. Omitola T, Gibbins N, Shadbolt N: Provenance in Linked Data Integration. Future Internet Assembly 2010, 1-8[http://eprints.ecs.soton.ac.uk/21954/]

21. Kessler C, Trame J, Kauppinen T: Tracking Editing Processes in Volunteered Geographic Information: The Case of OpenStreetMap. Processes and Events in Spatio-Temporally Distributed Data (IOPE), workshop at Conference on Spatial Information Theory 2011 (COSIT'11) 2011, 1-7[http:// www.carsten.io/iope2011.pdf].

22. Sahoo SS, Weatherly DB, Mutharaju R, Anantharam P, Sheth A, Tarleton RL: Ontology-Driven Provenance Management in eScience: An Application in Parasite Research. Proceedings of the Confederated International Conferences, CoopIS, DOA, IS, and ODBASE 2009 on On the Move to Meaningful Internet Systems: Part II, OTM '09 Berlin, Heidelberg: SpringerVerlag; 2009, 992-1009.

23. Missier P, Sahoo SS, Zhao J, Goble C, Sheth A: Janus: From workflows to Semantic Provenance and Linked Open Data. Procs IPAW 2010 2010, I(i):129-141.

24. Hull D, Wolstencroft K, Stevens R, Goble C, Pocock M, Li P, Oinn T: Taverna: a tool for building and running workflows of services. Nucleic Acids Res 2006, 34:W729-W732

25. Patni H, Sahoo SS, Henson C, Sheth A: Provenance Aware Linked Sensor Data. 2nd Workshop on Trust and Privacy on the Social and Semantic Web, Co-located with ESWC 2010, 1-12.

26. Cao B, Plale B, Subramanian G, Robertson E, Simmhan Y: Provenance Information Model of Karma Version 3. 3rd international workshop on scientific workflows SWF 2008, I(Vdl):348-351.

27. Marinho A, Werner C, da Cruz SMS, de Queirós Mattoso ML, Braganholo V, Murta L: A Strategy for Provenance Gathering in Distributed Scientific Workflows. Proceedings of the 2009 Congress on Services - I Washington, DC USA: IEEE Computer Society; 2009, 344-347[http://portal.acm.org/citation. cfm?id=1590963.1591573].

28. Coutinho F, Ogasawara E, de Oliveira D, Braganholo V, Lima AAB, D'avila AMR, Mattoso M: Data parallelism in bioinformatics workflows using Hydra. Proceedings of the 19th ACM International Symposium on High Performance Distributed Computing, HPDC '10 New York, NY, USA: ACM; 2010, 507-515.

29. Chapman A, Blaustein B, Elsaesser C: Provenance-based Belief. on the Theory and Practice of 2010, 1-14.

30. Braun UJ, Seltzer MI, Chapman A, Blaustein B, Allen MD, Seligman L: Towards Query Interoperability: PASSing PLUS. TaPP 2010, 1-10.

31. Gomes LSA: Provenance for bioinformatics workflows. Master's thesis Department of Informatics at Pontificial Catholic University, Rio de Janeiro, Brazil; 2011, [In Portuguese].

32. Gomes LA, Lifschitz S, Picouet P, Capriles PVSZ, Dardenne LE: A Provenance Model for Bioinformatics Workflows. Brazilian Symposium on Bioinformatics 2010, 19-22. 
33. Missier P, Belhajjame K: A PROV encoding for provenance analysis using deductive rules. Tech rep Newcastle University, Computing Science, Newcastle upon Tyne, NE1 7RU, England; 2012.

34. Ellson J, Gansner ER, Koutsofios E, North SC, Woodhull G: Graphviz and dynagraph - static and dynamic graph drawing tools. GRAPH DRAWING SOFTWARE Springer-Verlag; 2003, 127-148.

35. UNIPROT: Universal Protein Resource. 2012 [http://www.uniprot.org].

doi:10.1186/1471-2105-14-S11-S6

Cite this article as: de Paula et al.: Provenance in bioinformatics workflows. BMC Bioinformatics 2013 14(Suppl 11):S6.

Submit your next manuscript to BioMed Central and take full advantage of:

- Convenient online submission

- Thorough peer review

- No space constraints or color figure charges

- Immediate publication on acceptance

- Inclusion in PubMed, CAS, Scopus and Google Scholar

- Research which is freely available for redistribution 